\title{
New Rhodium(I) Carbene Complexes from Carbene Transfer
}

\section{Reactions}

\author{
Xiao-Yan Yu, Brian O. Patrick, and Brian R. James* \\ Department of Chemistry, The University of British Columbia, 2036 Main Mall, \\ Vancouver, British Columbia, Canada V6T $1 Z 1$
}

\section{Supporting Information}

Synthesis of $\mathrm{AgCl}(\mathrm{IPr}),\left[\mathrm{Ag}(\mathrm{IMes})_{2}\right] \mathrm{PF}_{6}$, and $\left[\mathrm{Ag}(\mathrm{IPr})_{2}\right] \mathrm{PF}_{6}$.

Table S1. Long C-H $\cdots$ F Contacts and Interaction Dimensions for $\left[\mathrm{Ag}(\mathrm{IMes})_{2}\right] \mathrm{PF}_{6}$ in $\AA$ and degree. (An asterisk indicates an intermolecular interaction. Estimated standard deviations are in parentheses)

Crystallographic data for complexes $\mathrm{AgCl}(\mathrm{IPr}),\left[\mathrm{Ag}(\mathrm{IMes})_{2}\right] \mathrm{PF}_{6}, \mathrm{RhCl}(\mathrm{NBD}(\mathrm{IMes})(\mathbf{1})$, and $\mathrm{RhCl}(\mathrm{COD})(\mathrm{MAI})(6)$ in $\mathrm{CIF}$ format.

\footnotetext{
* Corresponding author. Tel.: +1-(604)-822-6645; fax: +1-(604)-822-2847.

E-mail address: brj@chem.ubc.ca
} 
Syntheses. $\mathrm{AgCl}(\mathrm{IPr}) \cdot \mathrm{CH}_{2} \mathrm{Cl}_{2}(10 \mathrm{~mL})$ was added to a mixture of $\mathrm{Ag}_{2} \mathrm{O}(0.025 \mathrm{~g}$, $0.050 \mathrm{mmol})$ and $\mathrm{IPr} \cdot \mathrm{HCl}(0.045 \mathrm{~g}, 0.106 \mathrm{mmol})$, and the suspension was refluxed for 6 h. The resulting brownish suspension was cooled to room temperature and filtered through Celite. The brown filtrate was concentrated, and hexane $(10 \mathrm{~mL})$ was added to precipitate an off-white powder that was collected and dried in vacuo. Colorless crystals were obtained by dissolving the powder in $\mathrm{CH}_{2} \mathrm{Cl}_{2}(2 \mathrm{~mL})$, followed by addition of hexane (10 mL). Yield: $0.053 \mathrm{~g}(94 \%) .{ }^{1} \mathrm{H}$ NMR $\left(\mathrm{CDCl}_{3}\right): \delta 7.45(\mathrm{t}, 2 \mathrm{H}, J=7.8, p-\mathrm{CH})$, $7.25(\mathrm{~d}, 4 \mathrm{H}, J=7.8, m-\mathrm{CH}), 7.18(\mathrm{~d}, 2 \mathrm{H}, J=1.8, \mathrm{NCH}), 2.50(\mathrm{spt}, 4 \mathrm{H}, J=6.9$, $\left.\mathrm{CH}\left(\mathrm{CH}_{3}\right)_{2}\right), 1.23\left(\mathrm{~d}, 12 \mathrm{H}, J=6.9, \mathrm{CH}\left(\mathrm{CH}_{3}\right)_{2}\right), 1.18\left(\mathrm{~d}, 12 \mathrm{H}, J=6.9, \mathrm{CH}\left(\mathrm{CH}_{3}\right)_{2}\right) .{ }^{13} \mathrm{C}\left\{{ }^{1} \mathrm{H}\right\}$ NMR $\left(\mathrm{CDCl}_{3}\right): \delta 184.6\left(\mathrm{dd}, J\left({ }^{107} \mathrm{Ag}^{13} \mathrm{C}\right)=237, J\left({ }^{109} \mathrm{Ag}^{13} \mathrm{C}\right)=273, \mathrm{NCN}\right), 145.7(\mathrm{~s}, \mathrm{NC})$, 134.7 (s, NCC), 130.9 (s, $p$-CH), $124.5(\mathrm{~s}, m-C \mathrm{H}), 123.8\left(\mathrm{~d}, J\left({ }^{107} \mathrm{Ag}^{13} \mathrm{C}\right)=7, \mathrm{NCH}\right), 28.8$ (s, $\left.\mathrm{CH}\left(\mathrm{CH}_{3}\right)_{2}\right), 24.9$ (s, $\left.\mathrm{CH}\left(\mathrm{CH}_{3}\right)_{2}\right), 24.1$ (s, $\left.\mathrm{CH}\left(\mathrm{CH}_{3}\right)_{2}\right)$. ESI-MS (MeOH): 529, [M$\mathrm{Cl}+\mathrm{MeOH}]^{+}(51 \%) ; 497,[\mathrm{M}-\mathrm{Cl}]^{+}-(100 \%)$. Anal. Calcd for $\mathrm{C}_{27} \mathrm{H}_{36} \mathrm{~N}_{2} \mathrm{AgCl}: \mathrm{C}, 60.97 ; \mathrm{H}$, 6.82; N, 5.27. Found: C, 61.37; H, 7.02; N, 5.38.

[Ag(IMes) $\left.)_{2}\right]\left[\mathbf{P F}_{6}\right] \cdot \mathbf{M e O H}(10 \mathrm{~mL})$ was added to a mixture of $\mathrm{AgCl}(\mathrm{IMes})(0.090 \mathrm{~g}$, $0.200 \mathrm{mmol})$ and $\mathrm{NH}_{4} \mathrm{PF}_{6}(0.041 \mathrm{~g}, 0.251 \mathrm{mmol})$, and the suspension was stirred for 2 days at room temperature. The mixture was then evaporated to dryness and $\mathrm{CH}_{2} \mathrm{Cl}_{2}(10$ $\mathrm{mL}$ ) was added to give a yellowish suspension that was filtered through Celite; the filtrate was concentrated and addition of hexane $(10 \mathrm{~mL})$ precipitated an off-white powder that was collected and dried in vacuo. The compound was reprecipitated from $\mathrm{CH}_{2} \mathrm{Cl}_{2}$ by adding hexane (as for $\mathrm{AgCl}(\mathrm{IPr})$ ). Yield: $0.078 \mathrm{~g}$ (91\%, based on IMes). ${ }^{1} \mathrm{H}$ NMR $\left(\mathrm{CDCl}_{3}\right): \delta 7.05(\mathrm{~d}, 4 \mathrm{H}, J=2, \mathrm{NCH}), 6.87(\mathrm{~s}, 8 \mathrm{H}, m-\mathrm{Ar} H), 2.40\left(\mathrm{~s}, 12 \mathrm{H}, p-\mathrm{CH}_{3}\right), 1.68(\mathrm{~s}$, 
$\left.24 \mathrm{H}, o-\mathrm{CH}_{3}\right) .{ }^{31} \mathrm{P}\left\{{ }^{1} \mathrm{H}\right\}$ NMR $\left(\mathrm{CDCl}_{3}\right): \delta-143.2\left(\right.$ spt, $\left.P \mathrm{~F}_{6}{ }^{-}\right) .{ }^{13} \mathrm{C}\left\{{ }^{1} \mathrm{H}\right\}$ NMR $\left(\mathrm{CDCl}_{3}\right): \delta$ $182.6\left(\mathrm{dd}, J\left({ }^{107} \mathrm{Ag}^{13} \mathrm{C}\right)=180, J\left({ }^{109} \mathrm{Ag}^{13} \mathrm{C}\right)=208, \mathrm{NCN}\right), 139.4(\mathrm{~s}, \mathrm{NC}), 135.0(\mathrm{~s}, C-p-$ $\left.\mathrm{CH}_{3}\right), 134.7\left(\mathrm{~s}, C-o-\mathrm{CH}_{3}\right), 129.3(\mathrm{~s}, m-C \mathrm{H}), 123.2\left(\mathrm{~d}, J\left({ }^{107} \mathrm{Ag}^{13} \mathrm{C}\right)=6, \mathrm{NCH}\right), 21.3(\mathrm{~s}, p-$ $\mathrm{CH}_{3}$ ), 17.8 (s, o- $\left.\mathrm{CH}_{3}\right)$. ESI-MS (MeOH): 413, [M-IMes-PF $]^{+}$(100\%); 305, [IMes] ${ }^{+}$ (48\%). Anal. Calcd for $\mathrm{C}_{42} \mathrm{H}_{48} \mathrm{~N}_{4} \mathrm{AgPF}_{6}$ : C, 58.54; H, 5.61; N, 6.50. Found: C, 58.62; H, $5.48 ; \mathrm{N}, 6.27$.

$\left[\mathbf{A g}(\mathbf{I P r})_{2}\right]\left[\mathbf{P F}_{6}\right]$. This complex was prepared in a manner analogous to that described for $\left[\mathrm{Ag}(\mathrm{IMes})_{2}\right]\left[\mathrm{PF}_{6}\right]$, but using $0.053 \mathrm{~g}(0.100 \mathrm{mmol})$ of $\mathrm{AgCl}(\mathrm{IPr})$ and $0.019 \mathrm{~g}(0.110$ mmol) of $\mathrm{NH}_{4} \mathrm{PF}_{6}$. Yield: $0.043 \mathrm{~g}(83 \%$, based on $\mathrm{IPr}) .{ }^{1} \mathrm{H} \mathrm{NMR}\left(\mathrm{CDCl}_{3}\right): \delta 7.47(\mathrm{t}, 4 \mathrm{H}, J$ $=7.8, p-\mathrm{CH}), 7.13(\mathrm{~d}, 8 \mathrm{H}, J=7.8, m-\mathrm{CH}), 7.07(\mathrm{~d}, 4 \mathrm{H}, J=1.8, \mathrm{NCH}), 2.27(\mathrm{spt}, 8 \mathrm{H}, J=$ 6.9, $\left.\mathrm{CH}\left(\mathrm{CH}_{3}\right)_{2}\right), 1.06\left(\mathrm{~d}, 24 \mathrm{H}, J=6.9, \mathrm{CH}\left(\mathrm{CH}_{3}\right)_{2}\right), 0.77\left(\mathrm{~d}, 24 \mathrm{H}, J=6.9, \mathrm{CH}\left(\mathrm{CH}_{3}\right)_{2}\right)$. ${ }^{31} \mathrm{P}\left\{{ }^{1} \mathrm{H}\right\}$ NMR $\left(\mathrm{CDCl}_{3}\right): \delta$-143.2 (spt, $\left.P \mathrm{~F}_{6}{ }^{-}\right) .{ }^{13} \mathrm{C}\left\{{ }^{1} \mathrm{H}\right\}$ NMR $\left(\mathrm{CDCl}_{3}\right): \delta 183.6(\mathrm{dd}$, $\left.J\left({ }^{107} \mathrm{Ag}^{13} \mathrm{C}\right)=183, J\left({ }^{109} \mathrm{Ag}^{13} \mathrm{C}\right)=211, \mathrm{NCN}\right), 145.3(\mathrm{~s}, \mathrm{NC}), 134.6(\mathrm{~s}, \mathrm{NCC}), 129.9(\mathrm{~s}, p-$ $\mathrm{CH}), 125.0(\mathrm{~s}, m-\mathrm{CH}), 123.8\left(\mathrm{~d}, J\left({ }^{107} \mathrm{Ag}^{13} \mathrm{C}\right)=6, \mathrm{NCH}\right), 28.7\left(\mathrm{~s}, \mathrm{CH}\left(\mathrm{CH}_{3}\right)_{2}\right), 24.7(\mathrm{~s}$, $\left.\mathrm{CH}\left(\mathrm{CH}_{3}\right)_{2}\right), 24.0\left(\mathrm{~s}, \mathrm{CH}\left(\mathrm{CH}_{3}\right)_{2}\right)$. ESI-MS (MeOH): 497, [M-IPr-PF $]^{+}(100 \%) ; 389$, [IPr] ${ }^{+}$ (36\%). Anal. Calcd for $\mathrm{C}_{54} \mathrm{H}_{72} \mathrm{~N}_{4} \mathrm{AgPF}_{6}$ : C, 62.97; H, 7.05; N, 5.44. Found: C, 62.69; H, $7.15 ; \mathrm{N}, 5.27$. 
Table S1. Long C-H $\cdots$ F Contacts and Interaction Dimensions for $\left[\mathrm{Ag}(\mathrm{IMes})_{2}\right] \mathrm{PF}_{6}$ in $\AA$ and degrees. (An asterisk indicates an intermolecular interaction. Estimated standard deviations are in parentheses.)

\begin{tabular}{|c|c|c|c|c|c|}
\hline $\mathrm{C}-\mathrm{H} \cdots \mathrm{F}$ & $d(\mathrm{C}-\mathrm{H})$ & $d(\mathrm{H} \cdots \mathrm{F})$ & $d(\mathrm{C} \cdots \mathrm{F})$ & Angle $(\mathrm{CH} \cdots \mathrm{F})$ & C-Symmetry \\
\hline $\mathrm{C}(3)-\mathrm{H}(3) \cdots \mathrm{F}(2)$ & 0.95 & 2.38 & $2.990(6)$ & 122 & \\
\hline $\mathrm{C}(19)-\mathrm{H}(19 \mathrm{~b}) \cdots \mathrm{F}(5)$ & 0.98 & 2.48 & $3.400(7)$ & 156 & $2-\mathrm{x},-\mathrm{y},-\mathrm{z}$ \\
\hline $\mathrm{C}(24)-\mathrm{H}(24) \cdots \mathrm{F}(14)$ & 0.95 & 2.47 & $3.396(5)$ & 166 & \\
\hline $\mathrm{C}(31)-\mathrm{H}(31 \mathrm{c}) \cdots \mathrm{F}(11)$ & 0.98 & 2.54 & $3.476(6)$ & 160 & $\mathrm{x}, \mathrm{y},-1+\mathrm{z}$ \\
\hline $\mathrm{C}(44)-\mathrm{H}(44) \cdots \mathrm{F}(8)$ & 0.95 & 2.44 & $3.228(6)$ & 140 & $1-\mathrm{x},-\mathrm{y}, 1-\mathrm{z}$ \\
\hline $\mathrm{C}(44)-\mathrm{H}(44) \cdots \mathrm{F}(10)$ & 0.95 & 2.53 & $3.458(6)$ & 165 & $1-\mathrm{x},-\mathrm{y}, 1-\mathrm{z}$ \\
\hline $\mathrm{C}(57)-\mathrm{H}(57) \cdots \mathrm{F}(2)$ & 0.95 & 2.44 & $3.246(7)$ & 142 & $1-\mathrm{x},-\mathrm{y}, 1-\mathrm{z}$ \\
\hline $\mathrm{C}(65)-\mathrm{H}(65) \cdots \mathrm{F}(15)$ & 0.95 & 2.43 & $3.359(6)$ & 165 & $-1 / 2+x, 1 / 2-y, 1 / 2+z$ \\
\hline $\mathrm{C}(73)-\mathrm{H}(73 b) \cdots \mathrm{F}(12)$ & 0.98 & 2.53 & $3.422(6)$ & 152 & $-1+\mathrm{x}, \mathrm{y}, \mathrm{z}$ \\
\hline $\mathrm{C}(86)-\mathrm{H}(86) \cdots \mathrm{F}(7)$ & 0.95 & 2.47 & $3.368(6)$ & 156 & $-1 / 2+x, 1 / 2-y,-1 / 2+z$ \\
\hline $\mathrm{C}(90)-\mathrm{H}(90) \cdots \mathrm{F}(17)$ & 0.95 & 2.54 & $3.424(6)$ & 155 & $-1 / 2+x, 1 / 2-y,-1 / 2+z$ \\
\hline $\mathrm{C}(94)-\mathrm{H}(94 \mathrm{~b}) \cdots \mathrm{F}(17)$ & 0.98 & 2.53 & $3.454(7)$ & 157 & $-1 / 2+x, 1 / 2-y,-1 / 2+z$ \\
\hline $\mathrm{C}(101)-\mathrm{H}(101) \cdots \mathrm{F}(13)$ & 0.95 & 2.50 & $3.342(7)$ & 148 & $-1 / 2+x, 1 / 2-y, 1 / 2+z$ \\
\hline $\mathrm{C}(107)-\mathrm{H}(107) \cdots \mathrm{F}(3)$ & 0.95 & 2.44 & $3.204(6)$ & 138 & $1-\mathrm{x},-\mathrm{y},-\mathrm{z}$ \\
\hline $\mathrm{C}(107)-\mathrm{H}(107) \cdots \mathrm{F}(4)$ & 0.95 & 2.51 & $3.373(6)$ & 151 & $1-\mathrm{x},-\mathrm{y},-\mathrm{z}$ \\
\hline $\mathrm{C}(120)-\mathrm{H}(120) \cdots \mathrm{F}(16)$ & 0.95 & 2.45 & $3.309(7)$ & 151 & $-1 / 2+x, 1 / 2-y, 1 / 2+z$ \\
\hline
\end{tabular}

